\title{
Correction to: Minichromosome maintenance 3 promotes hepatocellular carcinoma radioresistance by activating the NF-kB pathway
}

Qing Yang ${ }^{1 \dagger}$, Binhui Xie ${ }^{2 \dagger}$, Hui Tang ${ }^{1 \dagger}$, Wei Meng ${ }^{1}$, Changchang Jia ${ }^{3}$, Xiaomei Zhang ${ }^{4}$, Yi Zhang $^{1}$, Jianwen Zhang ${ }^{1 *}$, Heping $\mathrm{Li}^{5^{*}}$ and Binsheng $\mathrm{Fu}^{1^{*}}$

\section{Correction to: J Exp Clin Cancer Res https://doi.org/10.1186/s13046-019-1241-9}

In the original publication of this article [1], the authors reported the order of the authors was incorrect and needs to be revised. The original article has been updated to rectify this error.

Incorrect version:

Wei Meng1, Binhui Xie2†, Qing Yang1†, Changchang Jia3, Hui Tang1†, Xiaomei Zhang4, Yi Zhang1, Jianwen Zhang1*, Heping Li5* and Binsheng Fu1*

Correct version:

Qing Yang1†, Binhui Xie2†, Hui Tang1†, Wei Meng1, Changchang Jia3, Xiaomei Zhang4, Yi Zhang1, Jianwen Zhang1*,Heping Li5* and Binsheng Fu1*

\footnotetext{
Author details

'Department of Hepatic Surgery and Liver transplantation Center of the Third Affiliated Hospital, Organ Transplantation Institute, Sun Yat-sen University, Organ Transplantation Research Center of Guangdong Province, 600\# Tianhe Road, Guangzhou 510630, China. ${ }^{2}$ Department of Hepatobiliary Surgery, The First Affiliated Hospital of Gannan Medical University, Ganzhou 341000, China. ${ }^{3}$ Cell-gene Therapy Translational Medicine Research Center, The Third Affiliated Hospital of Sun Yat-sen University, Guangzhou 510630, China. ${ }^{4}$ Guangdong Key Laboratory of Liver Disease Research, The Third Affiliated Hospital of Sun Yat-sen University, Guangzhou 510630, China. ${ }^{5}$ Department of Medical Oncology of the Eastern Hospital, The First Affiliated Hospital of Sun Yat-sen University, Zhongshan Er Road, Guangzhou 510080, China.
}

\footnotetext{
*Correspondence: zhjianw2@mail.sysu.edu.cn; drliheping@163.com; fubinsh@mail.sysu.edu.cn

${ }^{\dagger}$ Qing Yang, Binhui Xie and Hui Tang contributed equally to this work. 'Department of Hepatic Surgery and Liver transplantation Center of the Third Affiliated Hospital, Organ Transplantation Institute, Sun Yat-sen University, Organ Transplantation Research Center of Guangdong Province, 600\# Tianhe Road, Guangzhou 510630, China

${ }^{5}$ Department of Medical Oncology of the Eastern Hospital, The First Affiliated Hospital of Sun Yat-sen University, Zhongshan Er Road, Guangzhou 510080, China

Full list of author information is available at the end of the article
}

Published online: 02 August 2019

\section{Reference}

1. Yang $\mathrm{Q}$, et al. Minichromosome maintenance 3 promotes hepatocellular carcinoma radioresistance by activating the NF-KB pathway. J Exp Clin Cancer Res. 2019;38:263.

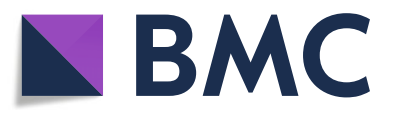

(อ) The Author(s). 2019 Open Access This article is distributed under the terms of the Creative Commons Attribution 4.0 International License (http://creativecommons.org/licenses/by/4.0/), which permits unrestricted use, distribution, and reproduction in any medium, provided you give appropriate credit to the original author(s) and the source, provide a link to the Creative Commons license, and indicate if changes were made. The Creative Commons Public Domain Dedication waiver (http://creativecommons.org/publicdomain/zero/1.0/) applies to the data made available in this article, unless otherwise stated. 